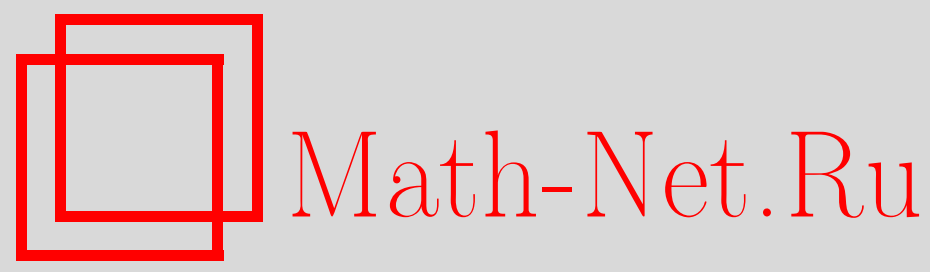

C. Н. Селезнева, О сложности задания $k$-значных функций обобщенно-поляризованными полиномами, Дискрет. матем., 2009, том 21, выпуск 4, 20-29

DOI: https://doi.org/10.4213/dm1068

Использование Общероссийского математического портала Math-Net.Ru подразумевает, что вы прочитали и согласны с пользовательским соглашением http://www.mathnet.ru/rus/agreement

Параметры загрузки:

IP : 54.89 .56 .158

26 апреля 2023 г., 08:41:51 


\title{
О сложности задания $k$-значных функций обобщенно-поляризованными полиномами
}

\author{
() 2009 г. С. Н. Селезнева
}

\begin{abstract}
Рассматриваются обобщенно-поляризованные полиномы $k$-значных функций (при простых $k$ ). Доказано, что по каждому вектору поляризации каждая $k$-значная функция задается однозначным обобщенно-поляризованным полиномом. Найдены верхняя и нижняя оценки функций Шеннона степени и длины обобщенно-поляризованных полиномов $k$-значных функций.

Работа выполнена при поддержке Российского фонда фундаментальных исследований, проекты 07-01-00444 и 09-01-00701а.
\end{abstract}

\section{1. Введение}

Одной из естественных форм задания функций являются полиномы. В зависимости от вида слагаемых рассматриваются обычные [1], поляризованные [2, 3], обобщенные [4, 5] полиномы и исследуется сложность задания функций такими полиномами.

В настоящей работе вводятся обобщенно-поляризованные полиномы для $k$-значных функций (при простых $k$ ) и находятся оценки сложности задания такими полиномами $k$-значных функций.

\section{2. Основные понятия}

Пусть $k \geqslant 2, E_{k}=\{0,1, \ldots, k-1\}$. Назовем $k$-значной функцией отображение $f^{n}: E_{k}^{n} \rightarrow E_{k}, n=0,1, \ldots$ Множество всех $k$-значных функций обозначим $P_{k}$, множество всех $k$-значных функций, зависящих от $n$ переменных, обозначим $P_{k}^{n}$.

Пусть $k$ - простое число. Будем рассматривать сложение и умножение по модулю $k$.

Произведение вида $x_{i_{1}}^{m_{1}} \ldots x_{i_{r}}^{m_{r}}$, в котором все переменные попарно различны, называется мономом. Степенью монома $X$ называется число

$$
\operatorname{deg}(X)=\sum_{j=1}^{r} m_{j} .
$$

Будем считать константу 1 вырожденным мономом степени 0.

Сумма вида $\sum_{i=1}^{l} c_{i} X_{i}$, где $c_{i} \in E_{k} \backslash\{0\}-$ коэффициенты, $X_{i}$ - различные мономы, $i=1, \ldots, l$, называется полиномом. Число слагаемых полинома $P$ называется его длиной $l(P)$, максимальная степень его слагаемых называется степенью $\operatorname{deg}(P)$ полинома. Мы 
будем полагать константу 0 вырожденным обобщенным полиномом с длиной и степенью, равными 0.

Известно, что каждую $k$-значную функцию можно однозначно задать полиномом по модулю $k$ тогда и только тогда, когда $k-$ простое число [1].

Введем понятие обобщенно-поляризованного полинома.

Обозначим $D_{m}, m=1, \ldots, k-1$. множество всех $k$-значных одноместных функций, степень которых равна $m$. Пусть

$$
\widetilde{D}=D_{k-1} \times \ldots \times D_{1}
$$

Обобщенным вектором поляризации назовем вектор $\delta=\left(\delta_{1}, \ldots, \delta_{n}\right)$, в котором $\delta_{i}=\left(s_{i, k-1}\left(x_{i}\right), \ldots, s_{i, 1}\left(x_{i}\right)\right) \in \widetilde{D}$, то есть степень полинома $s_{i, m}\left(x_{i}\right)$ равна $m$.

Обобщенно-поляризованным мономом по вектору $\delta$ называется произведение вида $\prod_{j=1}^{r} s_{i_{j}, m_{j}}\left(x_{i_{j}}\right)$, в котором все переменные попарно различны. Степенью обобщеннополяризованного монома $X$ называется число

$$
\operatorname{deg}(X)=\sum_{j=1}^{r} m_{j}
$$

Обобщенно-поляризованным полиномом по вектору $\delta$ назовем сумму различных обобщенно поляризованных по тому же вектору мономов с ненулевыми коэффициентами из $E_{k}$. Число слагаемых обобщенно-поляризованного полинома $P$ назовем его длиной $l(P)$, максимальную степень слагаемых - его степенью $d(P)$.

\section{3. О задании обобщенно-поляризованными полиномами $k$-значных функций}

Теорема 1. Пусть $k$ - простое число. Для каждого вектора поляризации $\delta$ для каждой $k$-значной функичи $f\left(x_{1}, \ldots, x_{n}\right)$ сущеествует однозначно определенный задающий ее обобщенно-поляризованный полином по этому вектору.

Доказательство. Вначале докажем существование для каждой функции задающего ее обобщенно-поляризованного полинома по каждому вектору поляризации.

Проведем индукцию по значению $n$.

В качестве базы индукции возьмем случай $n=1$. Пусть $\delta=\left(s_{k-1}(x), \ldots, s_{1}(x)\right)$, где $\operatorname{deg}\left(s_{i}\right)=i, i=k-1, \ldots, 1,-$ вектор поляризации.

Пусть дана функция $f(x) \in P_{k}^{1}$,

$$
f(x)=c_{k-1} x^{k-1}+\ldots+c_{1} x+c_{0},
$$

где $c_{k-1}, \ldots, c_{1}, c_{0} \in E_{k}$.

Запишем функцию $f(x)$ обобщенно-поляризованным полиномом по вектору $\delta$. Применим деление полиномов с остатком и получим

$$
\begin{array}{rlrl}
f(x) & =c_{k-1}^{\prime} s_{k-1}(x)+g_{k-2}(x), & & \operatorname{deg}\left(g_{k-2}\right) \leqslant k-2, \\
g_{k-2}(x) & =c_{k-2}^{\prime} s_{k-2}(x)+g_{k-3}(x), & & \operatorname{deg}\left(g_{k-2}\right) \leqslant k-2, \\
& \ldots, & & \\
g_{1}(x) c^{\prime} s_{1}(x) & =c_{0}, & c_{0}^{\prime}, c_{1}^{\prime}, \ldots c_{k-1}^{\prime} \in E_{k} .
\end{array}
$$


Тогда

$$
f(x)=c_{k-1}^{\prime} s_{k-1}(x)+\ldots+c_{1}^{\prime} s_{1}(x)+c_{0}^{\prime}
$$

есть обобщенно-поляризованный полином по вектору $\delta$ для функции $f(x)$.

Опишем индуктивный переход. Пусть для каждой функции от $n$ переменных найдется обобщенно-поляризованный по каждому вектору поляризации полином.

Рассмотрим функцию $f\left(y, x_{1}, \ldots, x_{n}\right) \in P_{k}^{n+1}$ :

$$
f\left(y, x_{1}, \ldots, x_{n}\right)=y^{k-1} f_{k-1}\left(x_{1}, \ldots, x_{n}\right)+\ldots+y f_{1}\left(x_{1}, \ldots, x_{n}\right)+f_{0}\left(x_{1}, \ldots, x_{n}\right) .
$$

Пусть $\delta=\left(\delta_{0}, \delta_{1}, \ldots, \delta_{n}\right)-$ вектор поляризации и $\delta^{\prime}=\left(\delta_{1}, \ldots, \delta_{n}\right)$. Учитывая предположение индукции, запишем каждую из функций $f_{k-1}\left(x_{1}, \ldots, x_{n}\right), \ldots, f_{1}\left(x_{1}, \ldots, x_{n}\right)$, $f_{0}\left(x_{1}, \ldots, x_{n}\right)$ обобщенно-поляризованным по вектору $\delta^{\prime}$ полиномом.

Затем, как при описании базиса индукции, проводя деление полиномов с остатком, поляризуем переменную $y$ по вектору $\delta_{0}$. При этом операции сложения и умножения на число с функциями от переменных $x_{1}, \ldots, x_{n}$ проводим формально над обобщеннополяризованными по вектору $\delta^{\prime}$ полиномами. Получим обобщенно-поляризованный полином по вектору $\delta$ для функции $f\left(y, x_{1}, \ldots, x_{n}\right)$.

Теперь докажем, что для каждой функции можно построить не более одного задающего ее обобщенно-поляризованного полинома по каждому вектору поляризации.

Пусть $\delta=\left(\delta_{1}, \ldots, \delta_{n}\right)$ - вектор поляризации. Подсчитаем число обобщенно-поляризованных по вектору $\delta$ полиномов от переменных $x_{1}, \ldots, x_{n}$.

Обобщенно-поляризованных по вектору $\delta$ мономов от переменных $x_{1}, \ldots, x_{n}$ можно составить $k^{n}$ (каждая переменная $x_{i}$ может либо не входить в моном, либо содержаться в нем в виде $\left.s_{i, m}\left(x_{i}\right), m=k-1, \ldots, 1\right)$. Из этих мономов можно построить $k^{k^{n}}$ обобщенно-поляризованных по вектору $\delta$ полиномов. Таким образом, число обобщеннополяризованных по вектору $\delta$ полиномов от переменных $x_{1}, \ldots, x_{n}$ совпадает с числом функций от этих же переменных.

Значит, каждая функция задается обобщенно-поляризованным полиномом по каждому вектору поляризации однозначно. Теорема 1 доказана.

Обобщенно-поляризованный полином для функции $f$ по вектору поляризации $\delta$ обозначим $P^{\delta}(f)$.

Заметим, что обобщенно-поляризованный полином для функции $f\left(x_{1}, \ldots, x_{n}\right)$ по вектору поляризации $\delta=\left(\left(x_{1}^{k-1}, \ldots, x_{1}\right), \ldots,\left(x_{n}^{k-1}, \ldots, x_{n}\right)\right)$ является обычным полиномом. Будем обозначать его $P(f)$.

Поляризованный полином из [3] для функции $f\left(x_{1}, \ldots, x_{n}\right) \in P_{k}^{n}$ по вектору поляризации $\left(d_{1}, \ldots, d_{n}\right) \in E_{k}^{n}$ является частным случаем обобщенно-поляризованного полинома по вектору поляризации $\delta=\left(\left(\left(x_{1}+d_{1}\right)^{k-1}, \ldots, x_{1}+d_{1}\right), \ldots,\left(\left(x_{n}+d_{n}\right)^{k-1}, \ldots, x_{n}+d_{n}\right)\right)$. Заметим, что для булевых функций обобщенно-поляризованные полиномы совпадают с поляризованными полиномами из [2].

\section{4. О сложности обобщенно-поляризованных полиномов}

Введем сложностные характеристики $k$-значных функций в классе обобщенно-поляризованных полиномов. Для функции $f\left(x_{1}, \ldots, x_{n}\right) \in P_{2}^{n}$ ее длиной $l^{G P P}(f)$ (степенью $\left.d^{G P P}(f)\right)$ в классе обобщенно-поляризованных полиномов назовем минимальную длину (минимальную степень) среди всех обобщенно-поляризованных полиномов, задающих $f$. 
Введем функции Шеннона длины и степени $k$-значных функций в классе обобщеннополяризованных полиномов, полагая

$$
L_{k}^{G P P}(n)=\max l^{G P P}(f), \quad D_{k}^{G P P}(n)=\max d^{G P P}(f),
$$

где максимум берется по всем функциям, зависящим от $n$ переменных.

Для функции Шеннона $L_{k}^{P P}(n)$ длины поляризованных полиномов по векторам поляризации $\left(d_{1}, \ldots, d_{n}\right) \in E_{k}^{n}$ известны следующие оценки:

(1) $L_{2}^{P P}(n)=\left\lfloor\frac{2}{3} 2^{n}\right\rfloor$ (cм. [2]),

(2) $L_{k}^{P P}(n) \leqslant \frac{k(k-1)}{k(k-1)+1} k^{n}$ при $k \geqslant 3$ (см. [3]),

(3) $\frac{k-1}{k} k^{n} \lesssim L_{k}^{P P}(n)$ при $n \rightarrow \infty$ (см. [6]),

(4) $L_{k}(1)=k-1$ (см. [7]).

Мы докажем следующие оценки функции Шеннона степени и длины обобщенно-поляризованных полиномов $k$-значных функций:

(1) $D_{k}^{G P P}(n)=(k-1) n$;

(2) $\frac{k-1}{k} k^{n} \lesssim L_{k}^{G P P}(n)$ при $n \rightarrow \infty$;

(3) $L_{k}^{G P P}(n) \leqslant \frac{k}{k+1} k^{n}$.

Заметим, что нижние мощностные оценки для поляризованных и обобщенно-поляризованных полиномов совпадают. Верхняя оценка обобщенно-поляризованных полиномов $k$-значных функций соответствует верхней оценке поляризованных полиномов булевых функций.

Вначале установим оценку степени обобщенно-поляризованных полиномов $k$-значных функций.

Теорема 2. Пусть $k$ - простое число. Тогда

$$
D_{k}^{G P P}(n)=(k-1) n
$$

Доказательство. Из доказательства теоремы 1 следует, что для каждой функции $f \in P_{k}$ для каждого вектора поляризации $\delta$ верно, что $\operatorname{deg}\left(P^{\delta}(f)\right)=\operatorname{deg}(P(f))$. Отсюда следует утверждение теоремы 2.

Теперь получим нижнюю и верхнюю оценки длины обобщенно-поляризованных полиномов $k$-значных функций.

Теорема 3. Пусть $k$ - простое число. Тогда

$$
\frac{k-1}{k} \lesssim L_{k}^{G P P}(n), \quad n \rightarrow \infty
$$


Доказательство. Пусть $L, L \leqslant(k-1) k^{n-1},-$ некоторое натуральное число. Найдем число обобщенно-поляризованных полиномов длины, не большей $L$, задающих функции от переменных $x_{1}, \ldots, x_{n}$.

Всего число мономов над переменными $x_{1}, \ldots, x_{n}$ есть $k^{n}$, полиномов длины, не большей $L$, из них можно составить $\sum_{i=0}^{L}\left(\begin{array}{c}k^{n} \\ i\end{array}\right)(k-1)^{i}$.

Каждую переменную можно обобщенно-поляризовать не более, чем $\left(k^{k}\right)^{(k-1)}$ способами (не более $k^{k}$ вариантов обобщенной поляризации для каждой степени переменной и $k-1$ степень переменной).

Следовательно, общее число обобщенно-поляризованных полиномов от переменных $x_{1}, \ldots, x_{n}$ длины, не большей $L$, не превосходит

$$
\sum_{i=0}^{L}\left(\begin{array}{c}
k^{n} \\
i
\end{array}\right)(k-1)^{i}\left(k^{k}\right)^{(k-1) n}
$$

Для оценки полученного выражения воспользуемся следующими свойствами.

1. Для всех целых $n \geqslant 1$ и $0 \leqslant i \leqslant n$ верно неравенство

$$
\left(\begin{array}{c}
n \\
i
\end{array}\right) \leqslant \frac{n^{n}}{i^{i}(n-i)^{n-i}}
$$

полагаем $0^{0}=1$.

Доказательство этого свойства можно провести индукцией по значению $n$.

2. Рассмотрим функцию действительного аргумента

$$
H_{k}(x)=x \log _{k} \frac{1}{x}+(1-x) \log _{k} \frac{1}{1-x}+x \log _{k}(k-1)
$$

на интервале $(0,1)$. Она называется функцией $k$-значной энтропии.

Несложно проверить, что

(1) $\lim _{x \rightarrow 0} H_{k}(x)=0, \quad \lim _{x \rightarrow 1} H_{k}(x)=\log _{k}(k-1)$,

(2) на полуинтервале $(0,(k-1) / k]$ функция $H_{k}(x)$ монотонно возрастает, на полуинтервале $[(k-1) / k, 1)$ функция $H_{k}(x)$ монотонно убывает, в точке $(k-1) / k$ она достигает своего максимума, и $H_{k}((k-1) / k)=1$.

Оценивая выражение (1) с использованием свойств 1 и 2 , находим, что

$$
\begin{aligned}
\sum_{i=0}^{L}\left(\begin{array}{c}
k^{n} \\
i
\end{array}\right)(k-1)^{i}\left(k^{k}\right)^{(k-1) n} & \leqslant L\left(\begin{array}{c}
k^{n} \\
L
\end{array}\right)(k-1)^{L} k^{k(k-1) n} \\
& \leqslant L \frac{\left(k^{n}\right)^{k^{n}}}{L^{L}\left(k^{n}-L\right)^{k^{n}-L}}(k-1)^{L} k^{k(k-1) n} \\
& \leqslant L k^{H_{k}\left(L / k^{n}\right) k^{n}} k^{k(k-1) n}
\end{aligned}
$$

Пусть $\varepsilon-$ положительная константа и $L \leqslant(k-1) / k-\varepsilon) k^{n}$. Тогда по свойствам функции $k$-значной энтропии найдется такая действительная положительная константа $\varepsilon_{1}$, что

$$
H_{k}\left(\frac{L}{k^{n}}\right) \leqslant H_{k}\left(\frac{k-1}{k}-\varepsilon\right) \leqslant 1-\varepsilon_{1} .
$$


Отсюда для выражения (2) находим, что

$$
L k^{H_{k}\left(L / k^{n}\right) k^{n}} k^{k(k-1) n} \leqslant\left(\frac{k-1}{k}-\varepsilon\right) k^{n} k^{\left(1-\varepsilon_{1}\right) k^{n}} k^{k(k-1) n} .
$$

Полученное выражение при $n \rightarrow \infty$ растет медленнее, чем $k^{k^{n}}$. Другими словами, для каждой действительной константы $\varepsilon>0$ при $L \leqslant((k-1) / k-\varepsilon) k^{n}$ обобщеннополяризованных полиномов длины, не большей $L$, не хватит, чтобы задать все функции от переменных $x_{1}, \ldots, x_{n}$. Отсюда следует, что при $n \rightarrow \infty$

$$
\frac{k-1}{k} k^{n} \lesssim L_{k}^{G P P}(n)
$$

Теорема 3 доказана.

Из теоремы 3 следует, что при задании $k$-значных функций обобщенно-поляризованными полиномами длину “самой сложной” функции асимптотически можно уменьшить не более, чем в константу раз.

Теорема 4. Пусть $k$ - простое число. Тогда

$$
L_{k}^{G P P}(n) \leqslant \frac{k}{k+1} k^{n}
$$

Доказательство. Доказательство проведем индукцией по числу переменных функции.

В качестве базиса индукции возьмем случай $n=1$. Рассмотрим $f(x) \in P_{k}^{1}$. Пусть $\operatorname{deg}(f)=m, m \geqslant 1$. Тогда длина обобщенно-поляризованного полинома функции $f(x)$ по вектору поляризации $\delta=\left(x^{k-1}, \ldots, x^{m+1}, f(x), x^{m-1}, \ldots, x\right)$ равна 1 .

Опишем индуктивный переход.

Пусть

$$
f\left(y, x_{1}, \ldots, x_{n}\right)=y^{k-1} f_{k-1}\left(x_{1}, \ldots, x_{n}\right)+\ldots+y f_{1}\left(x_{1}, \ldots, x_{n}\right)+f_{0}\left(x_{1}, \ldots, x_{n}\right) .
$$

По предположению индукции функцию $f_{k-1}\left(x_{1}, \ldots, x_{n}\right)$ можно так задать обобщеннополяризованным полиномом по некоторому вектору $\delta^{\prime}=\left(\delta_{1}, \ldots, \delta_{n}\right)$, что

$$
l\left(P^{\delta^{\prime}}\left(f_{k-1}\right)\right) \leqslant \frac{k}{k+1} k^{n} .
$$

Будем полагать, что все рассматриваемые дальше в доказательстве функции от переменных $x_{1}, \ldots, x_{n}$ записаны по вектору поляризации $\delta^{\prime}$.

Найдем такой вектор поляризации $\delta=\left(\delta_{0}, \delta_{1}, \ldots, \delta_{n}\right)$, в котором $\left(\delta_{1}, \ldots, \delta_{n}\right)=\delta^{\prime}$, что длина обобщенно-поляризованного полинома по нему функции $f\left(y, x_{1}, \ldots, x_{n}\right)$ не больше $k^{n+2} /(k+1)$.

В векторе $\delta$ неизвестна компонента $\delta_{0}$. Вектор $\delta_{0}$ построим последовательным уточнением.

Для уточнения вектора $\delta_{0}$ проведем последовательно шаги $1,2, \ldots, k-1$.

Опишем шаг $i, 1 \leqslant i \leqslant k-1$. Пусть на предыдущем шаге $i-1$ был получен вектор

$$
\delta_{0}^{i-1}=\left(s_{k-1}^{i-1}(y), \ldots, s_{(k-1)-(i-1)}^{i-1}(y), y^{(k-1)-i}, \ldots, y\right),
$$

вектор

$$
\delta^{i-1}=\left(\delta_{0}^{i-1}, \delta_{1}, \ldots, \delta_{n}\right)
$$


и функция

$$
\begin{aligned}
F_{i-1}\left(y, x_{1}, \ldots, x_{n}\right)= & s_{k-1}^{i-1}(y) f_{k-1}^{i-1}\left(x_{1}, \ldots, x_{n}\right) \\
& +\ldots \\
& +s_{(k-1)-(i-1)}^{i-1}(y) f_{(k-1)-(i-1)}^{i-1}\left(x_{1}, \ldots, x_{n}\right),
\end{aligned}
$$

для длины $L_{i-1}$ обобщенно-поляризованного полинома по вектору $\delta^{i-1}$ которой справедливо равенство

$$
L_{i-1}=\sum_{j=0}^{i-1} l\left(P^{\delta^{\prime}}\left(f_{(k-1)-j}^{i-1}\right)\right) \leqslant \beta_{i-1} k^{n},
$$

где $\beta_{i-1}-$ некоторая константа.

Если $i=1$, то положим

$$
\begin{aligned}
& \delta_{0}^{0}=\left(y^{k-1}, \ldots, y\right), \\
& \delta^{0}=\left(\delta_{0}^{0}, \delta_{1}, \ldots, \delta_{n}\right),
\end{aligned}
$$

и

$$
F_{0}\left(y, x_{1}, \ldots, x_{n}\right)=y^{k-1} f_{k-1}\left(x_{1}, \ldots, x_{n}\right)
$$

Заметим, что

$$
L_{0}=l\left(P^{\delta^{\prime}}\left(f_{k-1}\right)\right) \leqslant \frac{k}{k+1} k^{n}
$$

по предположению индукции. Поэтому положим $\beta_{0}=k /(k+1)$.

Выберем индекс $a, 0 \leqslant a \leqslant i-1$, так, что

$$
l_{i}=l\left(P^{\delta^{\prime}}\left(f_{(k-1)-a}^{i-1}\right)\right) \geqslant \frac{1}{i} L_{i-1} .
$$

Такой индекс $a$ всегда можно выбрать.

Пусть $X$ - произвольный обобщенно-поляризованный по вектору $\delta^{\prime}$ моном над переменными $x_{1}, \ldots, x_{n}$. Пусть $c^{\prime}$ и $c^{\prime \prime}-$ коэффициенты, с которыми он входит в полиномы $P^{\delta^{\prime}}\left(f_{(k-1)-a}^{i-1}\right)$ и $P^{\delta^{\prime}}\left(f_{(k-1)-i}\right)$ соответственно. Тогда, если $c^{\prime} \neq 0$, то

$$
c^{\prime} s_{(k-1)-a}^{i-1}(y) X+c^{\prime \prime} y^{(k-1)-i} X=c^{\prime}\left(s_{(k-1)-a}^{i-1}(y)+\frac{c^{\prime \prime}}{c^{\prime}} y^{(k-1)-i}\right) X .
$$

Пусть полиномы $g_{(k-1)-a}^{j}\left(x_{1}, \ldots, x_{n}\right)$ составлены из всех таких слагаемых $c^{\prime} X$, для которых $c^{\prime \prime} . c^{\prime}=j, j \in E_{k}$, а полином $g_{(k-1)-i}\left(x_{1}, \ldots, x_{n}\right)$ содержит все слагаемые полинома $P^{\delta^{\prime}}\left(f_{(k-1)-i}\right)$, которых нет в полиноме $P^{\delta^{\prime}}\left(f_{(k-1)-a}^{i-1}\right)$, со своими коэффициентами. Тогда

$$
\begin{aligned}
& s_{(k-1)-a}^{i-1}(y) f_{(k-1)-a}^{i-1}\left(x_{1}, \ldots, x_{n}\right)+y^{(k-1)-i} f_{(k-1)-i}\left(x_{1}, \ldots, x_{n}\right) \\
& =\sum_{j \in E_{k}}\left(s_{(k-1)-a}^{i-1}(y)+j y^{(k-1)-i}\right) g_{(k-1)-a}^{j}\left(x_{1}, \ldots, x_{n}\right)+y^{(k-1)-i} g_{(k-1)-i}\left(x_{1}, \ldots, x_{n}\right) .
\end{aligned}
$$

Заметим, что в полиномах $g_{(k-1)-a}^{j}\left(x_{1}, \ldots, x_{n}\right), j \in E_{k}, g_{(k-1)-i}\left(x_{1}, \ldots, x_{n}\right)$ по построению нет совпадающих слагаемых, может быть, с различными коэффициентами. Кроме того, по построению

$$
\sum_{j \in E_{k}} g_{(k-1)-a}^{j}\left(x_{1}, \ldots, x_{n}\right)=f_{(k-1)-a}^{i-1}\left(x_{1}, \ldots, x_{n}\right) .
$$


Выберем индекс $b \in E_{k}$ так, что

$$
l_{i}^{b}=l\left(P^{\delta^{\prime}}\left(g_{(k-1)-a}^{b}\right)\right) \geqslant \frac{1}{k} l_{i} .
$$

Такой индекс $b$ всегда можно выбрать.

Вектор поляризации $\delta_{0}^{i}$ получим из вектора поляризации $\delta_{0}^{i-1}$ заменой функции $s_{(k-1)-a}^{i-1}(y)$ на функцию $s_{(k-1)-a}^{i-1}(y)+b y^{(k-1)-i}$.

Переобозначим компоненты вектора $\delta_{0}^{i}$. Пусть

$$
\delta_{0}^{i}=\left(s_{k-1}^{i}(y), \ldots, s_{(k-1)-i}^{i}(y), y^{(k-1)-(i+1)}, \ldots, y\right) .
$$

Пусть $\delta^{i}=\left(\delta_{0}^{i}, \delta_{1}, \ldots, \delta_{n}\right)$ и

$$
F_{i}\left(y, x_{1}, \ldots, x_{n}\right)=F_{i-1}\left(y, x_{1}, \ldots, x_{n}\right)+y^{(k-1)-i} f_{(k-1)-i}\left(x_{1}, \ldots, x_{n}\right) .
$$

Запишем функцию $F_{i}\left(y, x_{1}, \ldots, x_{n}\right)$ по вектору поляризации $\delta^{i}$.

По рассмотренному выше получаем, что

$$
\begin{aligned}
& F_{i}\left(y, x_{1} \ldots, x_{n}\right)=F_{i-1}\left(y, x_{1}, \ldots, x_{n}\right)+y^{(k-1)-i} f_{(k-1)-i}\left(x_{1}, \ldots, x_{n}\right) \\
& =\sum_{j \in\{0,1, \ldots, i-1\}} s_{(k-1)-j}^{i-1}(y) f_{(k-1)-j}^{i-1}+y^{(k-1)-i} f_{(k-1)-i}\left(x_{1}, \ldots, x_{n}\right) \\
& =\sum_{j \in\{0,1, \ldots, i-1\} \backslash\{a\}} s_{(k-1)-j}^{i-1}(y) f_{(k-1)-j}^{i-1}\left(x_{1}, \ldots, x_{n}\right) \\
& +\left(s_{(k-1)-a}^{i-1}(y) f_{(k-1)-a}^{i-1}\left(x_{1}, \ldots, x_{n}\right)\right. \\
& \left.+y^{(k-1)-i} f_{(k-1)-i}\left(x_{1}, \ldots, x_{n}\right)\right) \\
& =\sum_{j \in\{0,1, \ldots, i-1\} \backslash\{a\}} s_{(k-1)-j}^{i-1}(y) f_{(k-1)-j}^{i-1}\left(x_{1}, \ldots, x_{n}\right) \\
& +\left(s_{(k-1)-a}^{i-1}(y)+b y^{(k-1)-i}\right) f_{(k-1)-a}^{i-1}\left(x_{1}, \ldots, x_{n}\right) \\
& +y^{(k-1)-i}\left(f_{(k-1)-i}\left(x_{1}, \ldots, x_{n}\right)-b f_{(k-1)-a}^{i-1}\left(x_{1}, \ldots, x_{n}\right)\right) \\
& =\sum_{j \in\{0,1, \ldots, i-1\} \backslash\{a\}} s_{(k-1)-j}^{i}(y) f_{(k-1)-j}^{i-1}\left(x_{1}, \ldots, x_{n}\right) \\
& +s_{(k-1)-a}^{i}(y) f_{(k-1)-a}^{i-1}\left(x_{1}, \ldots, x_{n}\right) \\
& +s_{(k-1)-i}^{i}(y)\left(f_{(k-1)-i}\left(x_{1}, \ldots, x_{n}\right)-b f_{(k-1)-a}^{i-1}\left(x_{1}, \ldots, x_{n}\right)\right) \text {. }
\end{aligned}
$$

Отсюда

$$
\begin{aligned}
f_{(k-1)-j}^{i}\left(x_{1}, \ldots, x_{n}\right) & =f_{(k-1)-j}^{i-1}\left(x_{1}, \ldots, x_{m}\right), \quad j=0,1, \ldots, i-1 \\
f_{(k-1)-i}^{i}\left(x_{1}, \ldots, x_{n}\right) & =f_{(k-1)-i}^{i-1}\left(x_{1}, \ldots, x_{m}\right)-b f_{(k-1)-a}^{i-1}\left(x_{1}, \ldots, x_{n}\right) .
\end{aligned}
$$

Для того, чтобы найти длину полинома $P^{\delta^{i}}\left(F_{i}\right)$ перепишем его в виде

$$
\begin{aligned}
& F_{i}\left(y, x_{1}, \ldots, x_{n}\right) \\
& =\sum_{j \in\{0,1, \ldots, i-1\} \backslash\{a\}} s_{(k-1)-j}^{i}(y) f_{(k-1)-j}^{i-1}\left(x_{1}, \ldots, x_{n}\right)+s_{(k-1)-a}^{i}(y) f_{(k-1)-a}^{i-1}\left(x_{1}, \ldots, x_{n}\right) \\
& \quad+s_{(k-1)-i}^{i}(y)\left(f_{(k-1)-i}\left(x_{1}, \ldots, x_{n}\right)-b f_{(k-1)-a}^{i-1}\left(x_{1}, \ldots, x_{n}\right)\right)
\end{aligned}
$$




$$
\begin{aligned}
& =\sum_{j \in\{0,1, \ldots, i-1\} \backslash\{a\}} s_{(k-1)-j}^{i}(y) f_{(k-1)-j}^{i-1}\left(x_{1}, \ldots, x_{n}\right) \\
& +s_{(k-1)-a}^{i}(y) \sum_{j \in E_{k}} g_{(k-1)-a}^{j}\left(x_{1}, \ldots, x_{n}\right) \\
& \quad+s_{(k-1)-i}^{i}(y)\left(\sum_{j \in E_{k} \backslash\{b\}} \tilde{g}_{(k-1)-a}^{j}\left(x_{1}, \ldots, x_{n}\right)+\tilde{g}_{(k-1)-i}\left(x_{1}, \ldots, x_{n}\right)\right),
\end{aligned}
$$

где полиномы $P^{\delta^{\prime}}\left(\tilde{g}_{(k-1)-a}^{j}\right)$ и $P^{\delta^{\prime}}\left(\tilde{g}_{(k-1)-i}\right)$ состоят из тех же слагаемых, что и соответственно полиномы $P^{\delta^{\prime}}\left(g_{(k-1)-a}^{j}\right)$ и $P^{\delta^{\prime}}\left(g_{(k-1)-i}\right)$, может быть, с другими коэффициентами.

Следовательно, полиномы

$$
P^{\delta^{\prime}}\left(g_{(k-1)-a}^{b}\right), \quad P^{\delta^{\prime}}\left(\tilde{g}_{(k-1)-a}^{j}\right), j \in E_{k} \backslash\{b\}, \quad P^{\delta^{\prime}}\left(\tilde{g}_{(k-1)-i}\right)
$$

не имеют общих слагаемых.

Таким образом,

$$
F_{i}\left(y, x_{1}, \ldots, x_{n}\right)=P_{1}+P_{2}+P_{3},
$$

где

$$
\begin{aligned}
P_{1}= & \sum_{j \in\{0,1, \ldots, i-1\} \backslash\{a\}} s_{(k-1)-j}^{i}(y) f_{(k-1)-j}^{i-1}\left(x_{1}, \ldots, x_{n}\right), \\
P_{2}= & s_{(k-1)-a}^{i}(y) g_{(k-1)-a}^{b}\left(x_{1}, \ldots, x_{n}\right) \\
& \quad+s_{(k-1)-i}^{i}(y)\left(\sum_{j \in E_{k} \backslash\{b\}} \tilde{g}_{(k-1)-a}^{j}\left(x_{1}, \ldots, x_{n}\right)+\tilde{g}_{(k-1)-i}\left(x_{1}, \ldots, x_{n}\right)\right), \\
P_{3}= & \sum_{j \in E_{k} \backslash\{b\}} s_{(k-1)-a}^{i}(y) g_{(k-1)-a}^{j}\left(x_{1}, \ldots, x_{n}\right) .
\end{aligned}
$$

Тогда, с учетом свойства (3),

$$
\begin{aligned}
l\left(P^{\delta^{i}}\left(F_{i}\right)\right) & \leqslant l\left(P_{1}\right)+l\left(P_{2}\right)+l\left(P_{3}\right) \\
& \leqslant\left(\beta_{i-1} k^{n}-l_{i}\right)+k^{n}+\frac{k-1}{k} l_{i} \leqslant \beta_{i-1} k^{n}+k^{n}-\frac{l_{i}}{k} \\
& \leqslant \beta_{i-1} k^{n}+k^{n}-\frac{1}{k i} \beta_{i-1} k^{n} \leqslant\left(1+\left(1-\frac{1}{k i}\right) \beta_{i-1}\right) k^{n} .
\end{aligned}
$$

Положим

$$
\beta_{i}=1+\left(1-\frac{1}{k i}\right) \beta_{i-1} .
$$

Заметим, что $F_{k-1}\left(y, x_{1}, \ldots, x_{n}\right)=f\left(y, x_{1}, \ldots, x_{n}\right)$.

Положим $\delta_{0}=\delta_{0}^{k-1}$ и $\delta=\left(\delta_{0}, \delta_{1}, \ldots, \delta_{n}\right)$.

Тогда обобщенно-поляризованный полином по вектору поляризации $\delta$ для функции $f\left(y, x_{1}, \ldots, x_{n}\right)$ имеет длину, не большую $\beta_{k-1} k^{n}$, которую можно подсчитать по следу- 
ющим равенствам при $\beta_{0}=k /(k+1)$ :

$$
\begin{gathered}
\beta_{1}=1+\left(1-\frac{1}{k}\right) \beta_{0}, \\
\beta_{2}=1+\left(1-\frac{1}{2 k}\right) \beta_{1}, \\
\cdots \\
\beta_{k-1}=1+\left(1-\frac{1}{(k-1) k}\right) \beta_{k-2} .
\end{gathered}
$$

В результате непосредственного подсчета получаем, что

$$
\beta_{k-1}=\frac{k^{2}}{k+1},
$$

или что

$$
l\left(P^{\delta}(f)\right) \leqslant \frac{k}{k+1} k^{n+1} .
$$

Индуктивный переход обоснован. Теорема 4 доказана.

Автор благодарит В. Б. Алексеева за ценные замечания, которые улучшили текст статьи.

\section{Список литературы}

1. Яблонский С. В., Введение в дискретную математику. Наука, Москва, 2001.

2. Перязев Н. А., Сложность булевых функций в классе полиномиальных поляризованных форм. Алгебра и логика (1995) 34, №3, 323-326.

3. Селезнева С. Н., О сложности представления функций многозначных логик поляризованными полиномами. Дискретная математика (2002) 14, №2, 48-53.

4. Кириченко К. Д., Верхняя оценка сложности полиномиальных нормальных форм булевых функций. Дискретная математика (2005) 17, №3, 80-88.

5. Селезнева С. Н., Дайняк А. Б., О сложности обобщенных полиномов $k$-значных функций. Becmник Московского Университета, сер. 15: вычисл. матем. и киберн. (2008) 34-39.

6. Алексеев В. Б., Вороненко А. А., Селезнева С. Н., О сложности реализации функций $k$-значной логики поляризованными полиномами. В сб.: Трудь V Международной конференции «Дискретные модели в теории управляющих систем». МГУ, Москва, 2003, с. 8-9.

7. Селезнева С. Н., О сложности поляризованных полиномов функций многозначных логик, зависящих от одной переменной. Дискретная математика (2004) 16, №2, 117-120.

Статья поступила 21.01.2009. 